\title{
Isotope Effect and Multiscale Physics in Fusion Plasmas
}

\author{
Y. Xu, ${ }^{1, *}$ C. Hidalgo, ${ }^{2}$ I. Shesterikov, ${ }^{1}$ A. Krämer-Flecken, ${ }^{3}$ S. Zoletnik, ${ }^{4}$ \\ M. Van Schoor, ${ }^{1}$ M. Vergote, ${ }^{1}$ and the TEXTOR Team \\ ${ }^{1}$ Laboratoire de Physique des Plasmas-Laboratorium voor Plasmafysica, Association "Euratom-Belgian state," \\ Ecole Royale Militaire-Koninklijke Militaire School, Trilateral Euregio Cluster, B-1000 Brussels, Belgium \\ ${ }^{2}$ Laboratorio Nacional de Fusion, Association EURATOM-CIEMAT, 28040 Madrid, Spain \\ ${ }^{3}$ Institute of Energy and Climate Research-Plasma Physics, Forschungszentrum Jülich GmbH, Association EURATOM-FZJ, \\ Trilateral Euregio Cluster, D-52425 Jülich, Germany \\ ${ }^{4}$ Institute for Particle and Nuclear Physics, Wigner Research Centre for Physics, Association EURATOM, \\ P.O. Box 49 H-1525, Budapest, Hungary
}

(Received 29 November 2012; published 26 June 2013)

\begin{abstract}
The mechanism governing the impact of the mass isotope on plasma confinement is still one of the main scientific conundrums facing the magnetic fusion community after more than thirty years of intense research. We have investigated the properties of local turbulence and long-range correlations in hydrogen and deuterium plasmas in the TEXTOR tokamak. Experimental findings have shown a systematic increasing in the amplitude of long-range correlations during the transition from hydrogen to deuterium dominated plasmas. These results provide the first direct experimental evidence of the importance of multiscale physics for unraveling the physics of the isotope effect in fusion plasmas.
\end{abstract}

DOI: 10.1103/PhysRevLett.110.265005

PACS numbers: 52.55.Fa, 52.25.Fi, 52.25.Gj, 52.35.Ra

Introduction.-There is clear experimental evidence that at comparable plasma discharge parameters deuterium (D) discharges have improved confinement properties as compared with hydrogen $(\mathrm{H})$ ones $[1,2]$. The isotope effect has been observed in many different tokamaks under different plasma conditions with a degree of confinement improvement in energy, particle, and momentum depending on plasma regimes. Interestingly, the isotope effect seems to be weaker, and eventually reversed, in stellarators than in tokamaks $[1,2]$. Understanding the physics of the isotope effect in plasma transport and confinement remains a fundamental open question confronting the fusion community since more than 30 years of intense research with direct impact in the confinement properties of fusion D-T reactors.

Considering that the characteristic step size of collisional transport and turbulent structures both increase with the plasma gyroradius $\rho_{s}$ [3], increasing the mass of the isotope would imply a deleterious effect on transport. Then assuming an estimation of the plasma diffusivity $\left(D_{0}\right)$ as the ratio of the square of a characteristic radial scale length $\left(L_{r}\right)$ over a characteristic time scale $\left(\tau_{c}\right), D_{0} \propto$ $L_{r}^{2} / \tau_{c}$, the isotope effect is a counterintuitive phenomenon if the typical radial length scales as $L_{r} \approx \rho_{s}$. In addition, while Bohm and gyro-Bohm behaviors are widely used to describe the empirical confinement time, they have, however, the wrong isotopic mass dependence.

Contemporary studies of transport phenomena in a wide range of research areas, including atmospheric flows [4], astrophysics [5], and fusion plasmas [6], have identified a common and fundamental feature of the physics of farfrom equilibrium systems-the "multiscale" physics, i.e., how large-scale structures can be developed by small-scale events. The breadth of these systems reveals the connectedness in natural sciences where the whole can be mirrored in the smallest parts. In magnetic fusion plasmas the largest scales are determined by the size of the device (in the order of meters), whereas the smallest scales are linked to the gyroradius of charge particles (in the order of $10^{-4} \mathrm{~m}$ for ions in the plasma edge).

Evidence of the importance of multiscale mechanisms for describing the properties of confinement in fusion plasmas has been accumulated both in plasma simulations and experimental results [7-10]. In particular, various experiments have shown the detection of long-range toroidal correlations consistent with the theory of zonal flows, i.e., large scale modes that are driven by turbulence and in return, regulate turbulent transport. The possible influence of the isotopic mass on shear flow rates [11] and zonal flows $[12,13]$ has also been proposed.

The main focus of this Letter is the empirical discovery of the amplification of long-range correlation (LRC) during the transition from $\mathrm{H}$ to $\mathrm{D}$ dominated plasmas, providing the first direct experimental evidence of the importance of multiscale physics in the description of the isotope effect in fusion plasmas. These findings suggest the role the ion mass on the amplitude of zonal-flow-like structures to unravel the ion mass dependence on transport and confinement in fusion plasmas.

Experimental setup.-Experiments were carried out in the TEXTOR tokamak [plasma minor radius $a \approx 0.48 \mathrm{~m}$, plasma major radius $R=1.75 \mathrm{~m}$, toroidal magnetic field $B_{T}=1.6-2.6 \mathrm{~T}$, plasma current $I_{p}=250-400 \mathrm{kA}$, and line-averaged plasma density $\bar{n}_{e}=(2.0-2.5) \times 10^{19} \mathrm{~m}^{-3}$ ] in Ohmically heated plasmas. The results reported here 

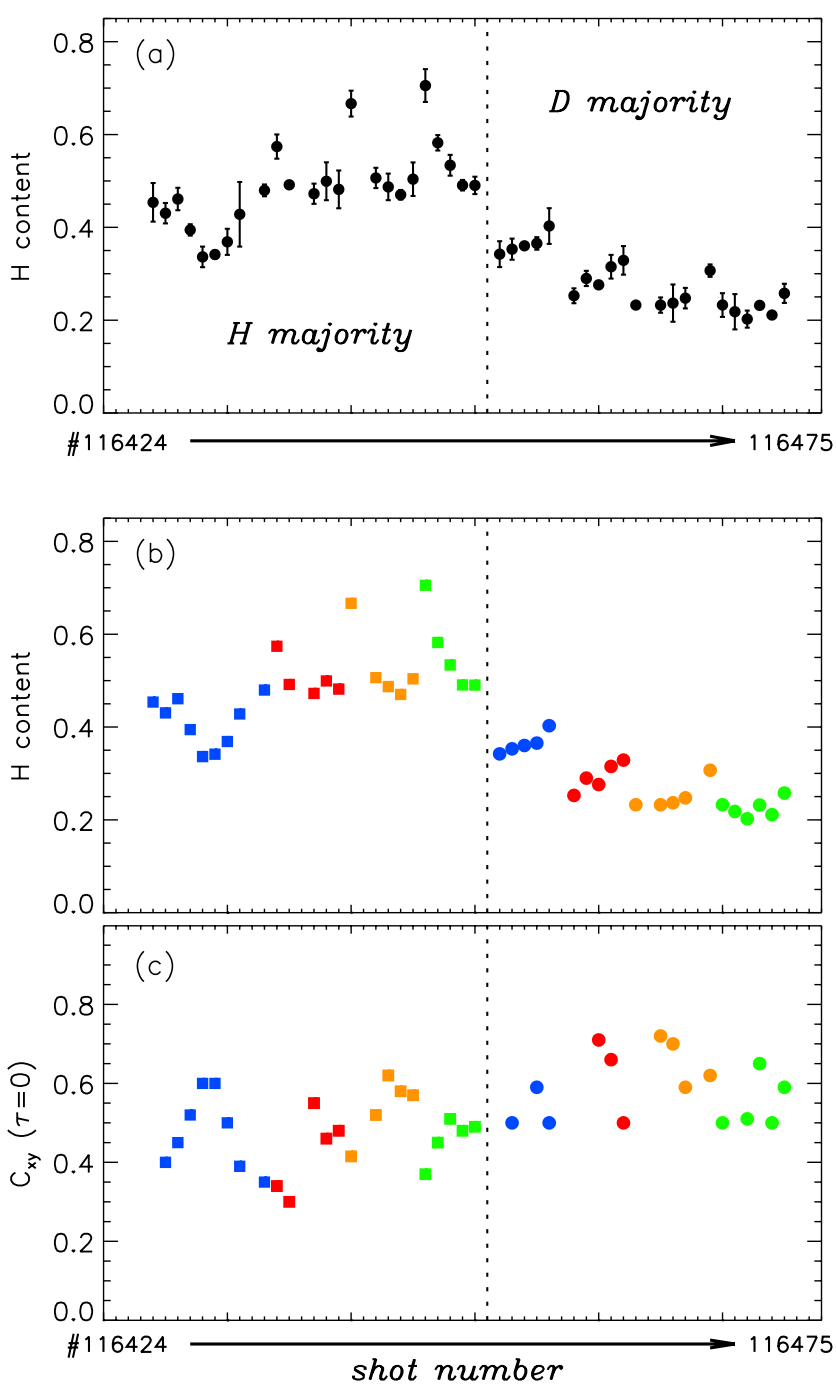

FIG. 1 (color online). Evolution of $\mathrm{H}$ content and long-range correlations versus plasma shot number at the TEXTOR tokamak: (a) $\mathrm{H}$ content, (b) $\mathrm{H}$ content highlighting shots with different $B_{T}$ conditions by four different colors (blue or dark gray, $2.25 \mathrm{~T}$, red or gray, $1.9 \mathrm{~T}$, yellow or light gray, $1.6 \mathrm{~T}$, green or medium gray, $2.6 \mathrm{~T}$ ), and (c) the maximum LRC values estimated by $C_{x y}(\tau=0)$. In (b) and (c) the squares and circles signify $\mathrm{H}$ and $\mathrm{D}$ dominated plasmas, respectively.

were mainly made by two toroidally spaced Langmuir probes arrays mounted at the outer midplane of the machine (one array is stationary and the other is a fast reciprocating system). The fast probe is moving from the scrape-off-layer into the plasma edge (three and a half centimeters inside the limiter radius), whereas the stationary probe stays at a fixed radial location in the plasma edge. This setup permitted the simultaneous investigation of short-range fluctuations (in the order of $1 \mathrm{~cm}$ due to broadband turbulence) and long-range fluctuating scales [in the order of $10 \mathrm{~m}$ due to geodesic-acoustic-mode (GAM) zonal flows], respectively, in the plasma boundary region of the TEXTOR tokamak $[8,14]$.

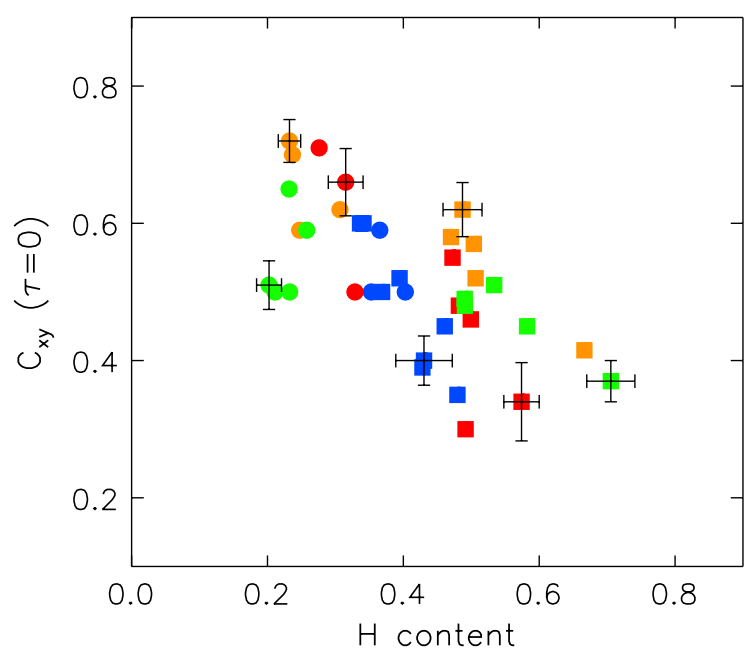

FIG. 2 (color online). Amplitude of the long-range correlation in floating potential signals as a function of $\mathrm{H}$ concentration for the whole shots shown in Fig. 1. The error bars on $C_{x y}(\tau=0)$ indicate the standard deviation about the mean measured in similar discharges.

The isotope effect has been previously investigated and compared in various experiments showing that the strength of the isotope effect is depending on plasma scenarios [1]. In the TEXTOR tokamak the ratio of the energy confinement time in $\mathrm{D}$ and $\mathrm{H}$ plasmas can reach values of 1.4 $[1,15]$. In this Letter, the H/D ratio was measured at the plasma edge on recycling light on the advanced limiter test (ALT) limiter. The observation is perpendicular to the ALT limiter blade 5. The light was transported via optics and light guides to a high resolution Echelle spectrometer which detected the Balmer-alpha line of $\mathrm{H}$ and $\mathrm{D}$. The lines were then fitted by a multiple line fit, providing the photon ( $\propto$ particle) flux ratio of H/D [16].

Experimental results and discussion.-In order to quantify the amplitude of long-range similarity of plasma fluctuations, we have computed the normalized cross correlation between floating potential signals, defined as [14]

$$
C_{x y}(\tau)=\frac{E\{[x(t)-\bar{x}][y(t+\tau)-\bar{y}]\}}{\sqrt{E\left\{[x(t)-\bar{x}]^{2}\right\} E\left\{[y(t)-\bar{y}]^{2}\right\}}}
$$

where $E$ is the expectation value and $\tau$ is the time lag. The two potential signals, $x(t)$ and $y(t)$, were measured at the above-mentioned two toroidal locations over a toroidal distance $\approx 7.0 \mathrm{~m}$.

In this study, to survey the isotope effect we have changed the $\mathrm{H}$ and $\mathrm{D}$ content during the experimental campaign by puffing different gas (H or D) species. In Fig. 1, the evolution of $\mathrm{H}$ content as well as the amplitude of long-range correlations between floating potential signals at zero time lag, i.e., the maximum LRC value denoted by $C_{x y}(\tau=0)$, are plotted versus shot numbers (from No. 116424 to No. 116475) during the transition from $\mathrm{H}$ to D dominated plasmas (separated by a vertical dashed 

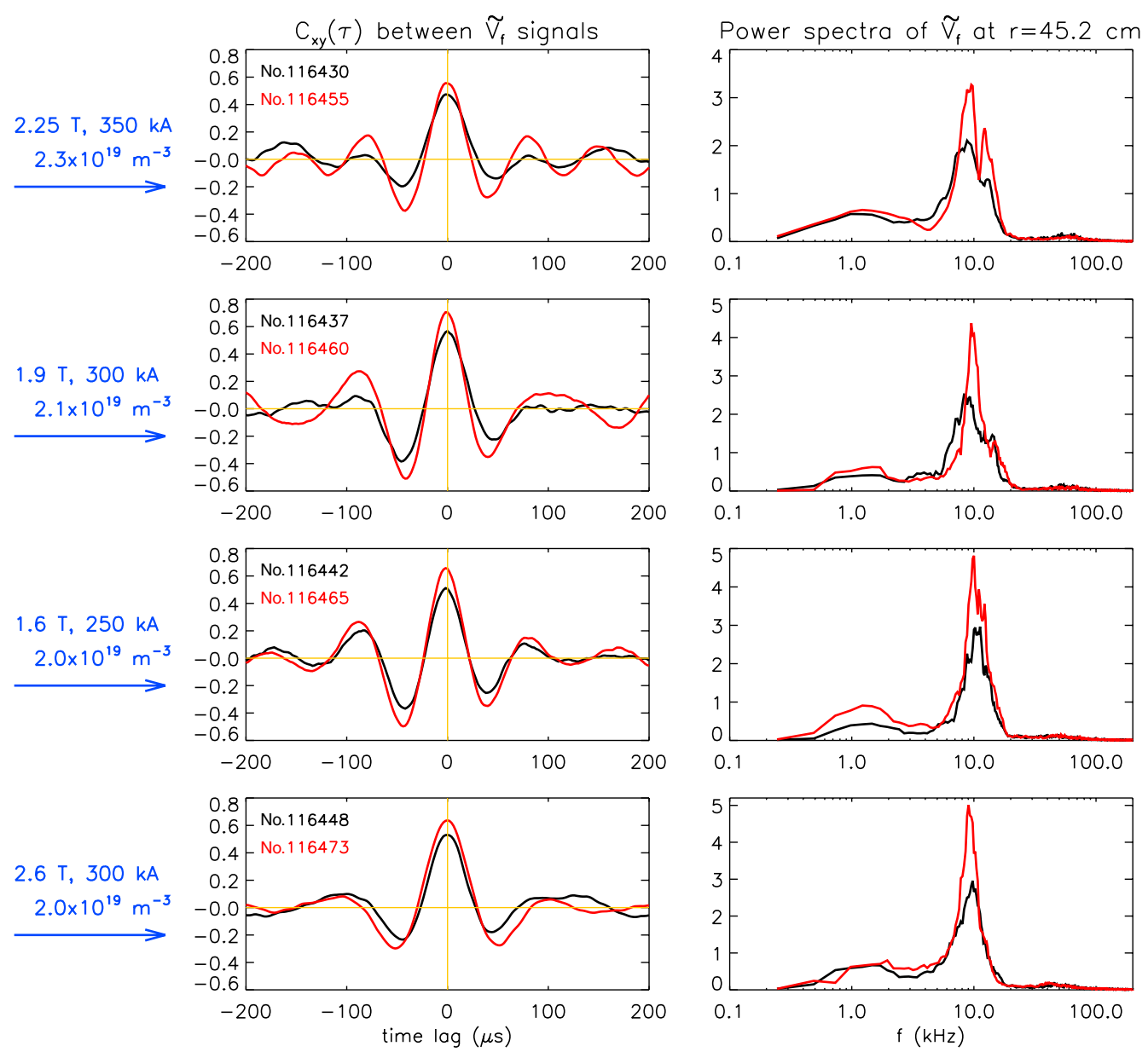

FIG. 3 (color online). Comparison of long-range cross-correlation functions (left column) and frequency spectra (right column) of floating potentials between $\mathrm{H}$ (black curves) and $\mathrm{D}$ (red curves) dominated plasmas in four same discharge conditions. The frequency spectra are detected at the maximum $C_{x y}$ location of $r \approx 45.2 \mathrm{~cm}$.

line). Measurements exploring four different magnetic fields $\left(B_{T}=2.25,1.9,1.6\right.$, and $\left.2.6 \mathrm{~T}\right)$ are marked with four different colors in Figs. 1(b) and 1(c), for both $\mathrm{H}$ majority (Nos. 116424-116450 shown by squares) and D majority (Nos. 116452-116475 shown by circles) plasmas. At each $B_{T}$, we scanned the plasma current and density shot by shot in the same range, i.e., $I_{p}=250-400 \mathrm{kA}$ and $\bar{n}_{e}=(2.0-2.5) \times 10^{19} \mathrm{~m}^{-3}$, so that we can make a good comparison on the isotopic behavior under similar plasma conditions. One notices that the smooth transition from $\mathrm{H}$ to D majority plasmas is accompanied with a corresponding smooth increasing in the amplitude of the long-range correlation. For each pair of $B_{T}$, the $\mathrm{D}$ dominated plasmas have relatively higher LRC values than $\mathrm{H}$ dominated ones. Figure 2 plots the amplitude of the long-range correlation in floating potential signals $C_{x y}(\tau=0)$ versus $\mathrm{H}$ concentration for the whole shots. It shows a systematic tendency with the LRC amplitude decreasing with increasing $\mathrm{H}$ concentration, regardless of plasma parameters.

Figure 3 further compares the long-range crosscorrelation function and the frequency spectra of floating potential fluctuations measured under four same discharge conditions (i.e., the same values of $B_{T}, I_{p}$, and $\bar{n}_{e}$ ) between $\mathrm{H}$ (black curves) and D (red curves) dominated plasmas. In all cases, the maximum of the long-range correlations is located close to the time delay zero and in amplitude of low frequency fluctuations, showing the long-range correlation is systematically larger in D dominated plasmas. It has been found that the observed long-range correlation is attributed to large-scale GAM zonal flows oscillating at $f \approx 10 \mathrm{kHz}$ with axisymmetric $n=0$ mode structures in potential fluctuations [14]. A similar increase in the LRC amplitude of GAM zonal flows has also been observed by the correlation reflectometer and Li-beam emission spectroscopy in density and velocity fluctuations at TEXTOR from $\mathrm{H}$ to D majority plasmas [17].

In order to study the local properties of plasma fluctuations we have computed the poloidal wave-number spectra $S\left(k_{\theta}\right)$ using the standard two-point correlation technique [18]. The two floating potential signals were measured by two poloidally separated pins $(5 \mathrm{~mm})$ of the fast probe. Figure 4 shows the comparison of $S\left(k_{\theta}\right)$ and frequency 

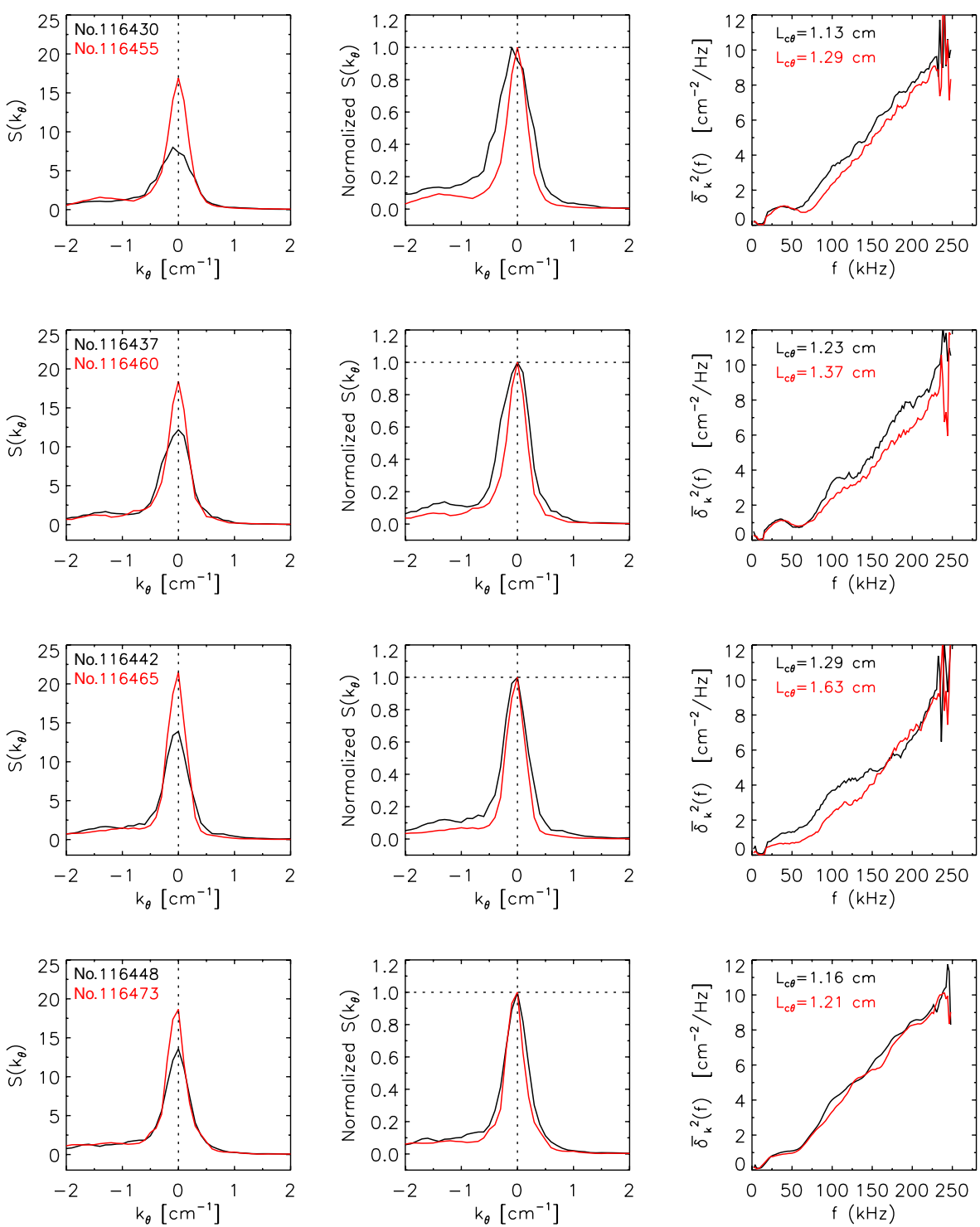

FIG. 4 (color online). Comparison of $S\left(k_{\theta}\right)$ and frequency resolved $k_{\theta}$-spectrum broadening, $\bar{\delta}_{k}^{2}(f)$, measured at $r \approx 45.2 \mathrm{~cm}$ between $\mathrm{H}$ (black curves) and D (red curves) majority plasmas, in four same discharge conditions of Fig. 3. Shown in the middle column are normalized $S\left(k_{\theta}\right)$ by their peaked values.

resolved $k$ spectrum broadening $\bar{\delta}_{k}^{2}(f)$ measured at $r \approx 45.2 \mathrm{~cm}$ between $\mathrm{H}$ (black curves) and $\mathrm{D}$ (red curves) majority plasmas under four same discharge conditions, which have been used to characterize the LRC and frequency spectrum $S(f)$ comparisons between $\mathrm{H}$ and $\mathrm{D}$ plasmas in Fig. 3. In the middle column, the $S\left(k_{\theta}\right)$ normalized by their peaked values shows a reduction in the spectrum width from black to red curves. Similarly, the $\bar{\delta}_{k}^{2}(f)$ is narrowed slightly but systematically from $\mathrm{H}$ to D dominated plasmas. In the right-column figures, the values of the poloidal correlation length $\left(L_{c \theta}\right)$ are estimated as the inverse of the $k$ spectrum broadening [18] and increase from $\mathrm{H}$ to $\mathrm{D}$ plasmas in all cases. Figure 5 depicts the evolution of the average poloidal correlation length versus $\mathrm{H}$ content in the whole plasma shot series. Results show that the $L_{c \theta}$ decreases with increasing $\mathrm{H}$ content; i.e., the poloidal correlation length increases during the smooth transition from $\mathrm{H}$ to $\mathrm{D}$ dominant plasmas, in agreement with previous experimental findings [3] as well as theoretical calculations [19].

The following mechanisms might explain the impact of the isotope effect on the amplitude of zonal-flow-like structures. The development of zonal flows by reversed energy cascades via $E \times B$ symmetry breaking mechanisms (i.e., eddy tilting) should be strongly sensitive to the size of turbulence structures [20,21]. Considering that the typical radial scale length of mean $E \times B$ sheared flows, $\lambda_{E \times B}$, is in the order of $1 \mathrm{~cm}$, the amplitude of inverse energy cascading processes is expected to be $k$ dependent with maximum energy transfer in $k$ scales in the order of $2 \pi / \lambda_{E \times B}$ (i.e., wave number in the range $5-10 \mathrm{~cm}^{-1}$ ). Then minor increasing of the size of turbulent 


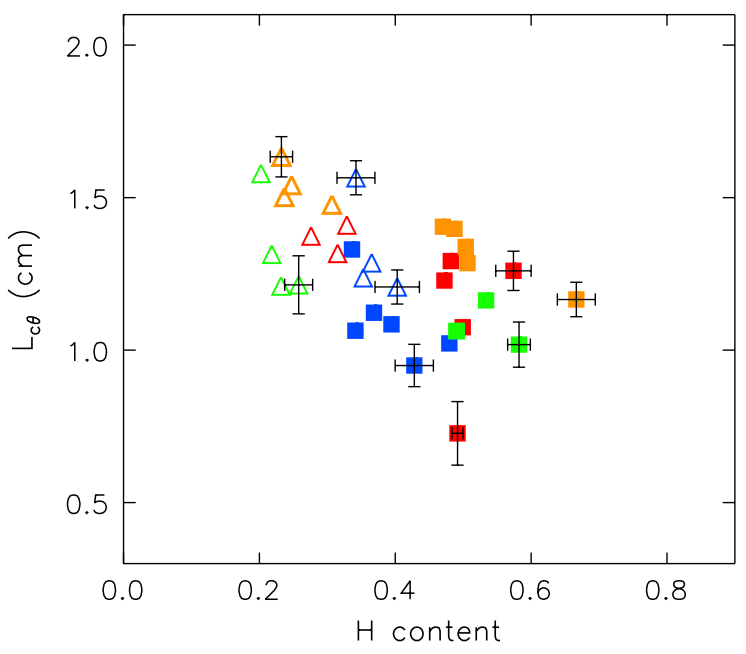

FIG. 5 (color online). Evolution of the poloidal correlation length $\left(L_{c \theta}\right)$ versus $\mathrm{H}$ concentration. The four different colors denote the four different $B_{T}$ discharges in Fig. 1. The filled squares and open triangles represent the $\mathrm{H}$ and $\mathrm{D}$ dominated plasmas, respectively. The error bars on $L_{c \theta}$ indicate the standard deviation about the mean measured in similar discharges.

structures $\left(\lambda_{\text {turb }}\right)$ in the range $\lambda_{\text {turb }} \approx \lambda_{E \times B}$ from light to heavy ion isotope (as those shown in Fig. 5) may eventually result in an increasing in the amplitude of zonal flows (Figs. 1-3) with beneficial effects on transport. In the framework of this interpretation, differences in the level of isotope effect in tokamaks and stellarators could be understood as a consequence of the stronger damping of zonal flows in nonoptimized stellarators than in tokamaks. However, other possibilities may also exist; e.g., the increases in zonal flows might be a result of better confinement of deuterium plasmas due to some other mechanisms caused by isotopic effects.

Conclusions.-We have investigated the properties of both local turbulence and large scale flows in hydrogen and deuterium plasmas in the TEXTOR tokamak. Experimental findings have shown a systematic increasing in the amplitude of zonal flows during the transition from $\mathrm{H}$ to $\mathrm{D}$ dominated plasmas. These results suggest the role the ion mass on the amplitude of zonal flows. These observations provide a guideline for further studies to unravel the physics of the isotope effect in fusion plasmas. The impact of the isotope effect on the energy transfer and selforganization mechanisms between turbulence and zonal flows should be addressed. In addition, the isotope effect would be expected to be stronger in magnetic configurations with reduced zonal flow damping.

The authors would like to thank the operation team for the dedicated experiments done at TEXTOR.

*Corresponding author. y.xu@fz-juelich.de

[1] M. Bessenrodt-Weberpals et al., Nucl. Fusion 33, 1205 (1993).

[2] U. Stroth, Plasma Phys. Controlled Fusion 40, 9 (1998).

[3] M. Ramisch, N. Mahdizadeh, U. Stroth, F. Greiner, C. Lechte, and K. Rahbarnia, Phys. Plasmas 12, 032504 (2005).

[4] V. Starr, Physics of Negative Viscosity Phenomena (McGraw-Hill, New York, 1968); P. Terry, Rev. Mod. Phys. 72, 109 (2000).

[5] R. Narayan and E. Quataert, Science 307, 77 (2005).

[6] P. H. Diamond, S.-I. Itoh, K. Itoh, and T. S. Hahm, Plasma Phys. Controlled Fusion 47, R35 (2005).

[7] A. Fujisawa, Nucl. Fusion 49, 013001 (2009).

[8] Y. Xu et al., Nucl. Fusion 51, 063020 (2011).

[9] C. Hidalgo et al., Europhys. Lett. 87, 55002 (2009).

[10] M. A. Pedrosa, C. Silva, C. Hidalgo, B. A. Carreras, R. O. Orozco, and D. Carralero, Phys. Rev. Lett. 100, 215003 (2008).

[11] X. Garbet, Plasma Phys. Controlled Fusion 39, B91 (1997).

[12] T. H. Watanabe, H. Sugama, and M. Nunami, Nucl. Fusion 51, 123003 (2011).

[13] T.S. Hahm, L. Wang, W.X. Wang, E. S. Yoon, and F. X. Duthoit, Nucl. Fusion 53, 072002 (2013).

[14] Y. Xu et al., Plasma Phys. Controlled Fusion 53, 095015 (2011).

[15] U. Samm et al., in Proceedings of the 16th EPS Conference on Plasma Physics, Venice, 1989 (European Physical Society, Geneva, 1989), Vol. 13B, Part III, p. 995.

[16] S. Brezinsek et al., Fusion Sci. Technol. 47, 209 (2005).

[17] A. Krämer-Flecken and S. Zoletnik (private communication).

[18] S. J. Levinson, J. M. Beall, E. J. Powers, and R. D. Bengtson, Nucl. Fusion 24, 527 (1984).

[19] M.Z. Tokar, D. Kalupin, and B. Unterberg, Phys. Rev. Lett. 92, 215001 (2004).

[20] A. Alonso et al., Plasma Phys. Controlled Fusion 48, B465 (2006).

[21] Y. Xu, C. Hidalgo, I. Shesterikov, M. Berte, P. Dumortier, M. Van Schoor, M. Vergote, A. Krämer-Flecken, R. Koslowski, and the TEXTOR Team, Nucl. Fusion 53, 072001 (2013). 